\title{
A generalisation of von Staudt's theorem on cross-ratios
}

\author{
YATIR HALEVI† AND ITAY KAPLAN $\ddagger$ \\ Einstein Institute of Mathematics, Edmond J. Safra Campus, The Hebrew University of \\ Jerusalem, Givat Ram 91904, Jerusalem, Israel. \\ e-mails: yatir.halevi@mail.huji.ac.il,kaplan@math.huji.ac.il \\ (Received 01 August 2018; revised 02 January 2019)
}

\begin{abstract}
A generalisation of von Staudt's theorem that every permutation of the projective line that preserves harmonic quadruples is a projective semilinear map is given. It is then concluded that any proper supergroup of permutations of the projective semilinear group over an algebraically closed field of transcendence degree at least 1 is 4-transitive.
\end{abstract}

2010 Mathematics Subject Classification: 14N99; 20B27; 20E28

\section{Introduction}

In his book Geometrie der Lage (see [vS47]), first appearing in 1847, Karl Georg Christian von Staudt, wanting to establish (real) projective geometry on an axiomatic approach, defined a projectivity to be a permutation of the projective line $\mathbb{P}(\mathbb{R})=\mathbb{R} \cup\{\infty\}$ preserving harmonic quadruples, i.e. quadruples of distinct elements having cross-ratio -1 (which can be defined strictly geometrically), where the cross-ratio of a quadruple of distinct elements is

$$
[a, b ; c, d]=\frac{c-a}{c-b} \cdot \frac{d-b}{d-a} .
$$

He proved that a projectivity is a composition of a finite number of perspectivities (which are basic geometric maps). It was noticed later that there is a small gap in von Staudt's reasoning, see [Coo34, Voe08] for a detailed historical background.

Given a field $F$, a projectivity (also known as a homography or a fractional linear transformation) of the projective line $\mathbb{P}(F)=F \cup\{\infty\}$ is an element of the group

$$
P G L_{2}(F)=\left\{\frac{a x+b}{c x+d}: a, b, c, d \in F, a d-b c \neq 0\right\},
$$

where the usual conventions when dealing with $\infty, 0$ and fractions apply here. It is easy to see that projectivities preserve cross-ratios.

$\dagger$ Supported by the European Research Council grant 338821, by ISF grant No. 181/16 and ISF grant No. $1382 / 15$.

$\$$ Supported by the Israel Science Foundation grants no. 1533/14 and 1254/18. 
It was Schreier and Sperner who first proved in [SS35, page 191] that every permutation of $F \cup\{\infty\}$, where $F$ is a field of characteristic $\neq 2$, preserving harmonic quadruples, i.e.

$$
[a, b ; c, d]=-1 \Longrightarrow[f(a), f(b) ; f(c), f(d)]=-1,
$$

is an element of

$$
P \Gamma L_{2}(F)=\left\{\frac{a x^{\sigma}+b}{c x^{\sigma}+d}: a, b, c, d \in F, a d-b c \neq 0, \sigma \in \operatorname{Aut}(F)\right\} .
$$

Any result in this spirit is now called a von Staudt theorem.

Over the years this theorem was generalised by relaxing the assumptions on $F$, for instance for $F$ a skew-field or a ring with some additional assumptions, see the introduction in [Hav15] for a survey of results in that direction.

In this paper, we follow a generalisation in a different direction. Hoffman eliminated the restriction on the characteristic of the field and replaced -1 with any field element which is fixed by $\operatorname{Aut}(F)$, see [Hof51]. Our main result is corollary $2 \cdot 16$ from the text:

THEOREM. Let $F$ be a field, $k$ its prime field and $\emptyset \neq O \subseteq F \backslash\{0,1\}$ which is Aut $(F)$ invariant. If

(i) $k(O) \subsetneq F$ and

(ii) if $\operatorname{char}(F)=2$ then $F$ is perfect and there exists an Aut $(F)$-invariant subfield $k \subsetneq L \subsetneq F$,

then the subgroup of permutations $f$ of $F \cup\{\infty\}$ satisfying

$$
[a, b ; c, d] \in O \Longleftrightarrow[f(a), f(b) ; f(c), f(d)] \in O
$$

is exactly $P \Gamma L_{2}(F)$.

The motivation for seeking such a generalisation came from infinite symmetric groups, and some model theory. It is well known that for a cardinal $\kappa$, the closed subgroups (in the product topology) of the infinite symmetric group $S_{\kappa}$, for a cardinal $\kappa$, in the product topology correspond exactly to automorphisms groups of first-order structures. Thus, finding closed supergroups of such groups sheds light on the the first order theory of such structures.

In [KS16], the second author and Pierre Simon proved that the affine groups $A G L_{n}(\mathbb{Q})$ (for $n \geq 2$ ) and the projective linear groups $P G L_{n}(\mathbb{Q})$ (for $n \geq 3$ ) are maximal closed in $S_{\omega}$. They ask whether it is true that $P \Gamma L_{2}(F)$ is maximal closed, for an algebraically closed field $F$ of transcendence degree greater that 1 . The aim of this paper is to step towards answering this question.

Bogomolov and Rovinsky proved that $P \Gamma L_{n}(F)$ is maximal closed for $n \geq 3$ and any field $F$, see [BR13]. The reason for the distinction between $n=2$ and $n \geq 3$ is that by the fundamental theorem of projective geometry, $P \Gamma L_{n}(F)$ (for $n \geq 3$ ) is exactly the collineation group of $\mathbb{P}^{n-1}(F)$. On the other hand, for $n=2$, since $\mathbb{P}^{1}(F)$ is the projective line, all the points are collinear.

If $P \Gamma L_{2}(F)$ were not maximal closed, a proper supergroup of it must preserve one out of a known family of relations, two of them being quaternary relations (see [KS16] for details). The aim is to show that it can not preserve any member of this family of relations. In this paper, using the above theorem, we conclude that if $F$ is an algebraically closed 
field of transcendence degree at least 1, then any group of permutation of $F \cup\{\infty\}$ properly containing $P \Gamma L_{2}(F)$ is 4-transitive and hence does not preserve any proper quaternary relation.

\section{Proofs}

Definition 2.1. Let $f$ be a permutation of $F \cup\{\infty\}$ and $\emptyset \neq O \subseteq F \backslash\{0,1\}$. We say that $f$ is $O$-preserving if

$$
[a, b ; c, d] \in O \Longleftrightarrow[f(a), f(b) ; f(c), f(d)] \in O,
$$

where $a, b, c, d$ are distinct elements from $F \cup\{\infty\}$.

Remark 2.2. In this paper the cross-ratio is only taken for distinct points so that it takes values in $F \backslash\{0,1\}$ (one can expand the definition to allow repetitions, but this will not be used).

Throughout we will implicitly use the following property of the cross-ratio:

For any two quadruples of distinct elements of $F \cup\{\infty\},\{A, B, C, D\}$ and $\{A, B, C, X\}$, the following holds:

$$
[A, B ; C, D]=[A, B ; C, X] \Longleftrightarrow D=X \text {. }
$$

Proposition 2.3. For every $x \in F \backslash\{0,1\}$ there exists a unique function

$$
g_{x}: X \longrightarrow F \cup\{\infty\}
$$

where $X \subseteq(F \cup\{\infty\})^{3}$ is the set of triples of distinct elements, such that for every distinct $a, b, c \in F \cup\{\infty\},\left[a, b ; c, g_{x}(a, b, c)\right]=x$. The function $g_{x}$ satisfies:

(i) $g_{x}(a, b, c) \neq a, b, c$ and

(ii) the map $x \mapsto g_{x}(a, b, c)$ is injective.

Furthermore, if $f$ is an $O$-preserving permutation of $F \cup\{\infty\}$, for some $\emptyset \neq O \subseteq F \backslash$ $\{0,1\}$, then for every distinct $a, b, c \in F \cup\{\infty\}$ there exists a permutation $\alpha: O \rightarrow O$, such for every $x \in O$

$$
f\left(g_{x}(a, b, c)\right)=g_{\alpha(x)}(f(a), f(b), f(c)) .
$$

Proof. The function $g_{x}$ is uniquely determined since, by the definition of the cross-ratio, we get the following formula:

$$
g_{x}(a, b, c)=\frac{b(c-a)-a x(c-b)}{(c-a)-x(c-b)} .
$$

Properties (i) and (ii) follow easily.

As for the furthermore, for $x \in O$, define

$$
\alpha(x):=\left[f(a), f(b) ; f(c), f\left(g_{x}(a, b, c)\right)\right] \in O
$$

and likewise

$$
\alpha^{-1}(x):=\left[a, b ; c, f^{-1}\left(g_{x}(f(a), f(b), f(c))\right)\right] \in O .
$$


They are both elements of $O$ since $f$ is $O$-preserving, and note that by uniqueness, the definition of $\alpha$ gives that

$$
f\left(g_{x}(a, b, c)\right)=g_{\alpha(x)}(f(a), f(b), f(c)) .
$$

Claim. For every $a, b, c, y \in F \cup\{\infty\}$ distinct, if $[a, b ; c, y] \in O$ then

$$
\alpha([a, b ; c, y])=[f(a), f(b) ; f(c), f(y)]
$$

and if $[f(a), f(b) ; f(c), y] \in O$ then

$$
\alpha^{-1}([f(a), f(b) ; f(c), y])=\left[a, b ; c, f^{-1}(y)\right] .
$$

Proof. Assume that $[a, b ; c, y]=x \in O$. By uniqueness necessarily

$$
y=g_{x}(a, b, c) .
$$

It now follows that

$$
\alpha([a, b ; c, y])=\alpha(x)=\left[f(a), f(b) ; f(c), f\left(g_{x}(a, b, c)\right)\right]=[f(a), f(b) ; f(c), f(y)],
$$

as required.

The proof for $\alpha^{-1}$ is similar.

We may now compute

$$
\begin{aligned}
\left(\alpha \circ \alpha^{-1}\right)(x) & =\alpha\left(\left[a, b ; c, f^{-1}\left(g_{x}(f(a), f(b), f(c))\right)\right]\right) \\
& =\left[f(a), f(b) ; f(c), g_{x}(f(a), f(b), f(c))\right]=x .
\end{aligned}
$$

Similarly we get that $\left(\alpha^{-1} \circ \alpha\right)(x)=x$, as needed.

Remark 2.4. The previous proposition is obviously also true if we permute the coordinates of the cross-ratio, e.g. consider a function $h_{x}$ which guarantees that

$$
\left[a, b ; h_{x}(a, b, c), c\right]=x .
$$

In Corollary 2.6, Lemma 2.7, Lemma 2.8 and Proposition 2.9 we work under the following assumption and notation:

ASSUMPTION 2.5. Let $O$ be a non-empty subset of $F \backslash\{0,1\}, f$ an $O$-preserving permu-

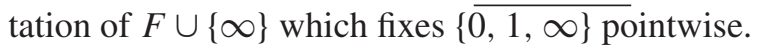

Furthermore, denote by $K:=k(O)$ the field generated by the elements of $O$, where $k$ is the prime field.

COROLlary 2.6. (Assumption 2.5) For all $a \neq b \in F$ there exist permutations $\tau_{a, b}, \rho_{a, b}$, $\chi_{a, b}, \alpha_{a, b}, \beta_{a, b}: O \rightarrow O$, such that for every $x \in O$ :

$$
\begin{gathered}
f(a x+b(1-x))=f(a) \tau_{a, b}(x)+f(b)\left(1-\tau_{a, b}(x)\right), \\
f\left(\frac{a-(1-x) b}{x}\right)=\frac{f(a)-\left(1-\rho_{a, b}(x)\right) f(b)}{\rho_{a, b}(x)},
\end{gathered}
$$




$$
\begin{gathered}
f\left(\frac{a-x b}{1-x}\right)=\frac{f(a)-\chi_{a, b}(x) f(b)}{1-\chi_{a, b}(x)} \\
f\left(\frac{a b x-b x-a b+a}{a x-x-b+1}\right)=\frac{f(a) f(b) \alpha_{a, b}(x)-f(b) \alpha_{a, b}(x)-f(a) f(b)+f(a)}{f(a) \alpha_{a, b}(x)-\alpha_{a, b}(x)-f(b)+1}, \\
f\left(\frac{a-b-a b x+b x}{a-b-a x+x}\right)=\frac{f(a)-f(b)-f(a) f(b) \beta_{a, b}(x)+f(b) \beta_{a, b}(x)}{f(a)-f(b)-f(a) \beta_{a, b}(x)+\beta_{a, b}(x)} .
\end{gathered}
$$

(For $\alpha_{a, b}$ and $\beta_{a, b}$ we also require that $a, b \neq 1$.)

Moreover, $f \uparrow O$ is a permutation of $O$.

Proof. We apply Proposition 2.3. Let $a \neq b \in F$. For $\tau_{a, b}$ use the identity

$$
[a x+b(1-x), a ; b, \infty]=x .
$$

For $\rho_{a, b}$ use the identity

$$
\left[a, \frac{a-(1-x) b}{x} ; b, \infty\right]=x .
$$

For $\chi_{a, b}$ use the identity

$$
\left[a, b ; \frac{a-x b}{1-x}, \infty\right]=x
$$

For $\alpha_{a, b}$ use the identity

$$
\left[b, a ; 1, \frac{a b x-b x-a b+a}{a x-x-b+1}\right]=x .
$$

For $\beta_{a, b}$ use the identity

$$
\left[b, 1 ; a, \frac{a-b-a b x+b x}{a-b-a x+x}\right]=x .
$$

In order to show that $f \uparrow O$ is a permutation of $O$, note that $[a, 1,0, \infty]=a$ for every $a \in F \backslash\{0,1\}$.

LEMMA 2.7. (Assumption 2.5) For every $a, b \in K$ and $x \in O$

$$
\begin{gathered}
f(a)+(1-x) f(b) \in f(K) \text { and } \\
x f(a)+f(b) \in f(K) .
\end{gathered}
$$

Proof. We start with the first assertion, so let $a, b \in K$ and $x \in O$. If $b=0$ there is nothing to show. If $a=0$ and $b \neq 0$, then since $\tau_{0, b}$ is a permutation, by Corollary $2 \cdot 6$,

$$
(1-x) f(b)=\left(1-\left(\tau_{a, b} \circ \tau_{a, b}^{-1}\right)(x)\right) f(b)=f\left(\left(1-\tau_{a, b}^{-1}(x)\right) b\right) \in f(K) .
$$

We may thus assume that $a, b \neq 0$ and let $x_{2}=\rho_{a, 0}^{-1}(x)$, so $f\left(a / x_{2}\right)=f(a) / x$. If $b=a / x_{2}$ then

$$
f(a)+f(b)-x f\left(\frac{a}{x_{2}}\right)=f(b) \in f(K) .
$$


Now, assume that $b \neq a / x_{2}$ and let $x_{3}=\tau_{a / x_{2}, b}^{-1}(x)$. Hence

$$
\begin{aligned}
f\left(\frac{a}{x_{2}} x_{3}+b\left(1-x_{3}\right)\right) & =f\left(\frac{a}{x_{2}}\right) \tau_{a / x_{2}, b}\left(x_{3}\right)+f(b)\left(1-\tau_{a / x_{2}, b}\left(x_{3}\right)\right) \\
& =f(a)+f(b)-x f(b) .
\end{aligned}
$$

Now the second assertion. If $a=0$ there is nothing to show. If $a \neq 0$ and $b=0$ then since $\tau_{a, b}$ is a permutation, $x f(a) \in f(K)$. We may thus assume that $a, b \neq 0$ and let $x_{2}=\chi_{b, 0}^{-1}(x)$. If $a=b /\left(1-x_{2}\right)$ then

$$
x f\left(\frac{b}{1-x_{2}}\right)+f(b)=\frac{x}{1-x} f(b)+f(b)=f(a) \in f(K) .
$$

Finally, assume that $a \neq b /\left(1-x_{2}\right)$, and let $x_{3}=\tau_{a, b /\left(1-x_{2}\right)}^{-1}(x)$. Hence

$$
\begin{aligned}
f\left(a x_{3}+\frac{b}{1-x_{2}}\left(1-x_{3}\right)\right) & =f(a) \tau_{a, b /\left(1-x_{2}\right)}\left(x_{3}\right)+f\left(\frac{b}{1-x_{2}}\right)\left(1-\tau_{a, b /\left(1-x_{2}\right)}\left(x_{3}\right)\right) \\
& =f(a) x+f(b) .
\end{aligned}
$$

Lemma 2.8. (Assumption 2.5) For every $0 \neq a \in K$ and $x \in O$,

$$
\begin{gathered}
-f(a)^{2} x+f(a) x+f(a) \in f(K) \text { and } \\
1+x-\frac{x}{f(a)} \in f(K) .
\end{gathered}
$$

Proof. Let $x \in O$ and $a \in K$ with $a \neq 0$. If $a=1$ both assertions are trivial, so assume $a \neq 1$.

We start with the first assertion. Since $\tau_{a, 0}$ is a permutation, by Corollary $2 \cdot 6$, we may define $x_{1}=\tau_{a, 0}^{-1}(x)$ so, $f\left(x_{1} a\right)=x f(a)$. We aim to use the permutation $\alpha_{a, x_{1} a}$. Obviously, $x_{1} a \neq a$ and if $x_{1} a=1$ then $x f(a)=1$ and $-f(a)^{2} x+f(a) x+f(a)=0$. Thus, by Corollary 2.6, $\alpha_{a, x_{1} a}$ is a permutation. Let $x_{2}:=\alpha_{a, x_{1} a}^{-1}(x)$, so

$$
\begin{aligned}
& f\left(\frac{a\left(x_{1} a\right) x_{2}-\left(x_{1} a\right) x_{2}-a\left(x_{1} a\right)+a}{a x_{2}-x_{2}-\left(x_{1} a\right)+1}\right) \\
& \quad=\frac{f(a) f\left(x_{1} a\right) x-f\left(x_{1} a\right) x-f(a) f\left(x_{1} a\right)+f(a)}{f(a) x-x-f\left(x_{1} a\right)+1}=-f(a)^{2} x+f(a) x+f(a) .
\end{aligned}
$$

Now for the second assertion. Since $f\left\lceil O\right.$ is a permutation, we may define $x_{1}:=f^{-1}(x)$. Note that, since $x \in O$, necessarily $x \neq 1$ and thus, since $f$ is permutation fixing $1, x_{1} \neq 1$ as well. We aim to use the permutation $\beta_{a, x_{1}}$. If $a=x_{1}$ then the statement is obviously true, so we may assume that $a \neq x_{1}$ (and both not equal to 1 ). By Corollary 2.6, $\beta_{a, x_{1}}$ is a permutation, so we may define $x_{2}:=\beta_{a, x_{1}}^{-1}(x)$ and so

$$
\begin{aligned}
f\left(\frac{a-x_{1}-a x_{1} x_{2}+x_{1} x_{2}}{a-x_{1}-a x_{2}+x_{2}}\right) & =\frac{f(a)-f\left(x_{1}\right)-f(a) f\left(x_{1}\right) x+f\left(x_{1}\right) x}{f(a)-f\left(x_{1}\right)-f(a) x+x} \\
& =1+x-\frac{x}{f(a)} .
\end{aligned}
$$

PROPOSITION 2.9. Using Assumption 2.5 and if when $\operatorname{char}(F)=2$ we assume further that $O$ is closed under taking square-roots, then $f(K)=K$. 
Proof. We first show that $K \subseteq f(K)$. Note that $O \subseteq f(K)$, indeed $f \uparrow O$ is a permutation by Corollary $2 \cdot 6$.

Let $a, b \in K$ and $x \in O$, which exists since $O$ is non-empty. By Lemma 2.7, $f(a)+$ $f(b)-x f(b) \in f(K)$. By the same lemma

$$
f(a)+f(b)=x f(b)+(f(a)+f(b)-x f(b)) \in f(K) .
$$

In order to show that if $f(c) \in f(K)$ then also $-f(c)$, first notice that by considering $\rho_{0, c}$ in Corollary 2.6 we see that

$$
-\frac{1-x}{x} f(c) \in f(K) .
$$

By considering $\chi_{d, 0}$ in the same corollary, we see that

$$
\frac{f(d)}{1-x} \in f(K)
$$

for any $d \in K$. In particular $-f(c) / x \in f(K)$, for any $c \in K$. Similarly, by considering $\tau_{a, 0}$, we get that $x f(c) \in f(K)$ for all $c \in K$ and together $-f(c) \in f(K)$ for all such $c$, as needed.

As for the multiplication, by Lemma $2 \cdot 8$,

$$
-f(a)^{2} x+f(a) x+f(a) \in f(K),
$$

for every $x \in O$. Using the above, and since $f(a), f(a) x \in f(K)$,

$$
-f(a)^{2} x \in f(K)
$$

So $-f(a)^{2} x=f(b)$ for some $b \in K$. Since $-f(b) / x \in f(K)$ as well, $f(a)^{2} \in f(K)$.

Let $a \neq 0 \in K$, by Lemma $2 \cdot 8,1+x-x / f(a) \in f(K)$ for any $x \in O$. Using the above, and since $1, x \in f(K)$,

$$
\frac{x}{f(a)} \in f(K)
$$

Using a similar argument to the previous paragraph, $-1 / f(a) \in f(K)$, so $1 / f(a) \in f(K)$.

Finally, we first assume that $\operatorname{char}(F) \neq 2$. Let $a, b \in K$. Since $(f(a)+f(b))^{2} \in f(K)$ we get that

$$
2 f(a) f(b) \in f(K) .
$$

To get that $f(K)$ is a subfield, we need this final claim:

Claim. If $a \in K$ then $f(a) / 2 \in f(K)$.

Proof. We may assume that $a \neq 0$. Since $f(a) \in f(K)$ then $1 / f(a) \in f(K)$ and so also $2 / f(a)$. Take the inverse again and $f(a) / 2 \in f(K)$.

We conclude that if $\operatorname{char}(F) \neq 2, f(K)$ is a field and so $K \subseteq f(K)$.

Assume that $\operatorname{char}(F)=2$. For any $n \geq 0$, let

$$
(f(K))^{2^{n}}:=\left\{a^{2^{n}}: a \in f(K)\right\} .
$$

Note that since $f(K)$ is closed under squares, $\left\{(f(K))^{2^{n}}: n \geq 0\right\}$ forms a decreasing sequence under inclusion. The following is an easy observation: 
Claim. For every $n \geq 0,(f(K))^{2^{n}}$ is also closed under addition, additive and multiplicative inverses and taking square powers.

Consider $L:=\bigcap_{n \geq 0}(f(K))^{2^{n}}$.

Claim. $L$ is a field containing $O$. Therefore, $K \subseteq L \subseteq f(K)$.

Proof. Since $O$ is closed under square-roots it is contained in $L$ and by the previous claim $L$ is closed under addition, additive and multiplicative inverses and taking square-powers. Let $a, b \in L$ and let $n \geq 0$. We will show that $a b \in(f(K))^{2^{n}}$. Since $a, b \in(f(K))^{2^{n+1}}$, by the following form of Hua's identity (first mentioned in [Hua49] but we use the more manageable form from [Jac68, page 2]):

$$
a-\left(a^{-1}+\left(b^{-2}-a\right)^{-1}\right)^{-1}=a^{2} b^{2}
$$

and, by the last claim, $a^{2} b^{2} \in(f(K))^{2^{n+1}}$. Since the Frobenius map is injective, $a b \in$ $(f(K))^{2^{n}}$, as required.

Either way, $K \subseteq f(K)$, but since $f^{-1}$ is also $O$-preserving by definition, we actually have $K \subseteq f(K) \subseteq K$ as required.

From now on we no longer assume Assumption 2.5.

Definition 2.10. For an $\operatorname{Aut}(F)$-invariant subfield $K \subseteq F$, a $K$-chain is an image of $K \cup\{\infty\}$ under the action of an element of $P \Gamma L_{2}(F)$.

Remark 2.11. The term $K$-chain is due originally to von Staudt who introduced it for any real subline of the complex projective line. Note that the usual definition of $K$-chain is for any subfield $K \subseteq F$ and only images under the action of $P G L_{2}(F)$ (see [Her95, definition 2.2.2]). Naturally, these definitions are equivalent for $A u t(F)$-invariant subfields. See [Her95] for more on this subject, and in a higher level of generality.

We recall that an action of a group $G$ on a set $X$ (with $|X| \geq k$ ) is $k$-transitive if $G$ acts transitively on the set of $k$-tuples of distinct elements of $X$. For example, the action of the group $P G L_{2}(F)$ on $F \cup\{\infty\}$ is 3-transitive.

COROLLARY 2.12. Let $f$ be an $O$-preserving permutation of $F \cup\{\infty\}$, for some non-empty $O \subseteq F \backslash\{0,1\}$ which is Aut $(F)$-invariant. If, when $\operatorname{char}(F)=2$, we further assume $O$ is closed under taking square-roots, then $f$ sends $K$-chains to $K$-chains, where $K=k(O)$.

Proof. Let $X=T(K \cup\{\infty\})$ be a $K$-chain, where $T \in P \Gamma L_{2}(F)$. Since $O$ is $\operatorname{Aut}(F)$ invariant, $f \circ T$ is also $O$-preserving. Thus we may assume that $X=K \cup\{\infty\}$. Since $P G L_{2}(F)$ is 3-transitive and preserves the cross-ratio, by composing with an element of $P G L_{2}(F)$ we may assume that $f$ fixes $\{0,1, \infty\}$ pointwise. By Proposition 2.9, $f(K \cup$ $\{\infty\})=K \cup\{\infty\}$ as needed.

We recall some definitions from affine geometry. Given a $K$-vector space $V$, an affine line is a set of the form $K a+b$ for some $a, b \in V$ with $a \neq 0$. A map $T: V \rightarrow V$ is called 
semilinear if there exists a field automorphism $\sigma \in \operatorname{Aut}(K)$ such that for all $v, u \in V$ and $x, y \in K$

$$
T(x v+y u)=\sigma(x) T(v)+\sigma(y) T(u) .
$$

FACT 2.13 (The Fundamental Theorem of Affine Geometry). [BR98, theorem 3.5.6] Let $K$ be any field and $V$ a $K$-vector space of dimension at least 2. If $f$ a permutation of $V$ sending affine lines to affine lines then there exists a semilinear map $T: V \rightarrow V$ and $b \in V$ such that $f(x)=T(x)+b$ for all $x \in V$.

The following theorem is a generalisation of the main theorem in [Hof51].

THEOREM 2.14. Let $k \subsetneq F$ be a field, where $k$ is the prime field of $F$, and let $f$ be a permutation of $F \cup\{\infty\}$.

If $\operatorname{char}(F)=2$ we assume further that:

(a) $F$ is perfect and

(b) there exists an Aut (F)-invariant subfield $k \subsetneq L \subsetneq F$.

Then the following are equivalent:

(i) $f \in P \Gamma L_{2}(F)$;

(ii) $f$ is $O$-preserving for all non-empty $O \subseteq F \backslash\{0,1\}$ which are Aut $(F)$-invariant and satisfy $k(O) \subsetneq F$;

(iii) $f$ is $O$-preserving for some non-empty $O \subseteq F \backslash\{0,1\}$ which is Aut $(F)$-invariant and satisfies $k(O) \subsetneq F$;

(iv) There exists an Aut $(F)$-invariant subfield $K \subsetneq F$ such that $f$ sends $K$-chains to K-chains;

(v) For all Aut $(F)$-invariant subfields $K \subsetneq F, f$ sends $K$-chains to $K$-chains.

Remark $2 \cdot 15$. The implication (iv) $\Rightarrow$ (i) is well known for fields $F$ with $\operatorname{char}(F) \neq 2$, see [Her95, theorem 9.2.5], but we provide a direct proof.

Proof. (i) $\Rightarrow$ (ii). This is by the definition of $P \Gamma L_{2}(F)$.

(ii) $\Rightarrow$ (iii). If $\operatorname{char}(F) \neq 2$ just take $O=k \backslash\{0,1\}$ (which is non-empty). If $\operatorname{char}(F)=2$, then take $O=L \backslash\{0,1\}$, where $L$ is the given $\operatorname{Aut}(F)$-invariant subfield.

(iii) $\Rightarrow$ (iv). Let $K=k(O)$. Since $O$ is $\operatorname{Aut}(F)$-invariant, $K$ is $\operatorname{Aut}(F)$-invariant and if $\operatorname{char}(F)=2$ then $O$ is closed under taking square-roots since $F$ is perfect and hence the inverse of the Frobenius map is an automorphism. Now apply Corollary $2 \cdot 12$.

(iv) $\Rightarrow$ (i). By composing with an element of $P G L_{2}(F)$, we may assume that $f$ fixes $\{0,1, \infty\}$ pointwise. We plan to use Fact $2 \cdot 13$, so we must show that, in $F$ as a $K$-vector space, $f \uparrow F$ sends affine lines to affine lines. Since $f$ fixes $\{\infty\}$, and sends $K$-chains to $K$-chains, it is sufficient to show the following, where by a projective affine line we mean a union of an affine line with $\{\infty\}$,

Recall also that any $K$-chain is equal to some $T(K \cup\{\infty\})$, where $T(x)$ is of the form

$$
\frac{a x^{\sigma}+b}{c x^{\sigma}+d},
$$

for $a, b, c, d \in F$, with $a d-b c \neq 0$, and $\sigma \in \operatorname{Aut}(F)$. Since $K$ is Aut $(F)$-invariant we may assume that $\sigma=i d$. 
Claim. A subset of $F \cup\{\infty\}$ is a $K$-chain which includes $\infty$ if and only if it is a projective affine line.

Proof. A projective affine line has the form $a(K \cup\{\infty\})+b$, for $a, b \in F$, so it is a $K$-chain. For the other direction, by translation it is enough to show that any $K$-chain containing 0 and $\infty$ is a projective affine line.

Note that projective affine lines that contain 0 are just of the form $a K$ (for $a \neq 0$ ), and that both families of projective affine lines containing 0 and $K$-chains containing 0 and $\infty$ are closed under scalar multiplication (by non-zero elements from $F$ ) and inverse $(x \mapsto 1 / x)$. So it is enough to show that after applying finitely many operations of the form above on a $K$-chain containing 0 and $\infty$ gives a projective affine line (containing 0 ). Assume that we are given a $K$-chain of the form $T(K \cup\{\infty\})$ for $T$ as above which contains 0 and $\infty$.

(i) If $T$ is of the form $a x+b$ with $a \neq 0$, then $b=0$ and we are done.

(ii) If $T$ is of the form $b /(c x+f)$ with $c \neq 0$, then we are done by the first bullet (after dividing by $b$ and taking inverse).

(iii) If the $T$ is of the form $(a x+b) /(c x+d)$ with $a, c \neq 0$, then after multiplying by $c / a$, we may assume that $a=c=1$, and then since the chain contains $0, b \in K$, and since the chain contains $\infty, d \in K$. So it is equal to $(K \cup\{\infty\})$.

As a result, $f$ preserves the system of affine lines in the $K$-vector space $F$. Since $K \subsetneq F, \operatorname{dim}_{K} F \geq 2$ so by the fundamental theorem of affine geometry (Fact 2.13) and since $f(0)=0, f$ must be additive and so also $f(-a)=-f(a)$ for all $a \in F$.

The conjugation of $f$ by the $P G L_{2}(F)$ map $x \mapsto 1 / x$ also satisfies the above, so it is also additive. This translates to

$$
\frac{f(a) f(b)}{f(a)+f(b)}=f\left(\frac{a b}{a+b}\right)
$$

for all nonzero $a, b \in F$. By setting in the equation $a=1$ and $b=t-1$ (for $t \neq 1$ ) we obtain, $f(t) f\left(t^{-1}\right)=1$, thus $f$ commutes with inversion.

Putting in the same equation $b=1-a$, for $a \neq 0,1$, we obtain $f(a) f(1-a)=$ $f(a(1-a))$, which gives $f\left(a^{2}\right)=f(a)^{2}$.

If $\operatorname{char}(F) \neq 2$ then, since $f$ is additive, $f(x / 2)=f(x) / 2$ for all $x \in F$. Set $a=x+y$ in the last equation to get $f(x y)=f(x) f(y)$ for all $x, y \in F$.

If $\operatorname{char}(F)=2$ we once again use Hua's identity:

$$
\begin{gathered}
f\left(a^{2} b^{2}\right)=f\left(a-\left(a^{-1}+\left(b^{-2}-a^{-1}\right)^{-1}\right)\right. \\
=f(a)-\left(f(a)^{-1}+\left(f(b)^{-2}-f(a)^{-1}\right)^{-1}\right)=f\left(a^{2}\right) f\left(b^{2}\right) .
\end{gathered}
$$

Hence $f(a b)^{2}=(f(a) f(b))^{2}$, so $f(a b)=f(a) f(b)$.

Either way, we get that $f$ is an automorphism of $F$, an in particular $f \in P \Gamma L_{2}(F)$.

(i) $\Rightarrow$ (v) is clear and (v) $\Rightarrow$ (iv) follows by taking $K=k$.

As a direct corollary of Theorem $2 \cdot 14$ we get the following. 
COROLlary 2-16. Let $F$ be a field, $k$ its prime field and $\emptyset \neq O \subseteq F \backslash\{0,1\}$ which is Aut $(F)$-invariant. If:

(i) $k(O) \subsetneq F$ and

(ii) if $\operatorname{char}(F)=2$ then $F$ is perfect and there exists an Aut $(F)$-invariant subfield $k \subsetneq$ $L \subsetneq F$,

then the subgroup of $O$-preserving permutations of $F \cup\{\infty\}$ is exactly $P \Gamma L_{2}(F)$.

It was shown by Hoffman in [Hof51], that if $F$ is a field, $a \in F \backslash\{0,1\}$ and $f$ is an $\{a\}$-preserving permutation of $F \cup\{\infty\}$ then $f \in P \Gamma L_{2}(F)$. One may ask, what about if $f$ preserves a set of cardinality larger than 1 ? Theorem $2 \cdot 14$ only gives a partial answer. More specifically, can the proper containment of fields assumptions be dropped? Can the perfectness assumption when $\operatorname{char}(F)=2$ be dropped? For example:

Question 2.17. Does the subgroup of permutations of $\mathbb{Q}(\sqrt{2}) \cup\{\infty\}$ which are $\{ \pm \sqrt{2}\}$ preserving properly contain $P \Gamma L_{2}(\mathbb{Q}(\sqrt{2}))$ ?

\section{Every proper extension of $P \Gamma L_{2}(F)$ is 4-transitive}

Our final aim is to show that, as a corollary of Theorem $2 \cdot 14$, any group of permutations of $F \cup\{\infty\}$, for $F$ algebraically closed of transcendence degree at least 1, which properly contains $P \Gamma L_{2}(F)$ must be 4-transitive and as a result does not preserve any non-trivial 4-relation.

LEMMA 3.1. Let $\left\{O_{i}\right\}_{i \in I}$ be the orbits of Aut $(F)$ acting on $F \backslash\{0,1\}$. Then the orbits of $P \Gamma L_{2}(F)$ acting on quadruples of distinct elements from $F \cup\{\infty\}$ are

$$
\left\{(a, b, c, d):[a, b ; c, d] \in O_{i}\right\}_{i \in I} .
$$

Proof. Let $T \circ \sigma \in P \Gamma L_{2}(F$,$) , for T \in P G L_{2}(F)$ and $\sigma \in \operatorname{Aut}(F)$. Since elements of $P G L_{2}(K)$ preserve the cross-ratio, and $\sigma\left(O_{i}\right)=O_{i}$ by definition, $P \Gamma L_{2}(F)$ preserves the orbits.

Now, let $(x, y, z, w),(a, b, c, d)$ be quadruples of distinct elements such that

$$
[x, y ; z, w],[a, b ; c, d] \in O_{i} .
$$

By applying an element of $\operatorname{Aut}(F)$ we may assume that $[x, y ; z, w]=[a, b ; c, d]$.

Since $P G L_{2}(F)$ is 3-transitive, there exists $T \in P G L_{2}(K)$ such that $T(x)=a$, $T(y)=b, T(z)=c$. So we have that

$$
[a, b ; c, T(w)]=[a, b ; c, d] .
$$

As $a, b, c$ are distinct we have that $T(w)=d$.

THEOREM 3.2. Let $F$ be an algebraically closed field of transcendence degree at least 1 , and $H$ be a group of permutations of $F \cup\{\infty\}$ properly containing $P \Gamma L_{2}(F)$. Then $H$ is 4-transitive.

Proof. By Lemma 3.1, the action of $P \Gamma L_{2}(F)$ breaks the space of quadruples of distinct elements from $F \cup\{\infty\}$ into infinitely many finite orbits (corresponding to finite Galois orbits) and one infinite orbit (corresponding to the Galois orbit of transcendentals). 
Thus it is enough to show that every orbit of the action of $H$ on the space of quadruples of distinct elements from $F \cup\{\infty\}$ intersects the orbit corresponding to the transcendentals.

Aiming for a contradiction, assume there exists an orbit $X$ of the action of $H$, which only contains orbits with algebraic cross-ratio, i.e.

$$
X=\bigcup_{i \in I_{0}}\left\{(a, b, c, d):[a, b ; c, d] \in O_{i}\right\},
$$

for some $I_{0} \subseteq I$ and $O_{i}$ finite, where $I$ and $O_{i}$ are as in Lemma 3.1. Let $O=\bigcup_{i \in I_{0}} O_{i}$ be the cross-ratios arising from quadruples from $X$ and let $K=k(O)$, where $k$ is the prime field. Note that $K \subsetneq F$ and that $O$ is $\operatorname{Aut}(F)$ invariant. By assumption every element of $H$ is $O$-preserving and thus by Corollary 2.16, $H \subseteq P \Gamma L_{2}(F)$, contradiction.

Question 3.3. What about other fields? For instance, is it true that every group of permutations of $\mathbb{Q}(\sqrt{2}) \cup\{\infty\}$ properly containing $P \Gamma L_{2}(\mathbb{Q}(\sqrt{2}))$ must be 4-transitive?

Acknowledgements. This project started as a derivative from the work of the second author with Pierre Simon in [KS16]. Eventually ideas from that time contributed to the proof of the main theorem here, namely in the proof of (iv) implies (i) in Theorem $2 \cdot 14$. We thank him for allowing us to use his ideas. We also thank the anonymous referees for their careful reading, their comments and for pointing out a small error in a previous formulation of the main theorem.

\section{REFERENCES}

[BR98] A. BEUtelsPaCHER and U. RosenbaUm. Projective geometry: from foundations to applications (Cambridge University Press, Cambridge, 1998).

[BR13] F. BOGOMOLOV and M. Rovinsky. Collineation group as a subgroup of the symmetric group. Cent. Eur. J. Math., 11(1) (2013), 17-26.

[Coo34] J. L. Coolidge. The rise and fall of projective geometry. Amer. Math. Monthly 41(4):1934, 217-228.

[Hav15] H. HAVLICEK. Von Staudt's theorem revisited. Aequationes Math. 89(3) (2015), 459-472.

[Her95] A. HERZER. Chain geometries. In Handbook of Incidence Geometry, pages 781-842 (NorthHolland, Amsterdam, 1995).

[Hof51] A. J. Hoffman. A note on cross ratio. Amer. Math. Monthly 58 (1951), 613-614.

[Hua49] L.-K. HuA. Some properties of a sfield. Proc. Natl. Acad. Sci., 35(9) (1949), 533-537.

[Jac68] N. JACOBSON. Structure and representations of Jordan algebras. Amer. Math. Soc. Colloq. Publ., vol. XXXIX (American Mathematical Society, Providence, R.I., 1968).

[KS16] I. KAPLAN and P. SimON. The affine and projective groups are maximal. Trans. Amer. Math. Soc, 368(7) (2016), 5229-5245.

[SS35] O. SCHREIER and E. SPERNER. Einführung in die Analytische Geometrie und Algebra, volume 2 (BG Teubner, 1935).

[Voe08] J.-D. VOELKE. Le théorème fondamental de la géométrie projective: évolution de sa preuve entre 1847 et 1900. Arch. Hist. Exact Sci. 62(3) (2008), 243-296.

[vS47] K. G. C. von Staudt. Geometrie der Lage (Verlag von Bauer und Raspe (Julius Merz), Nürnberg, 1847). 